\title{
Development of Objective Index to Determine Autism by Eyeball Movements
}

\author{
Ippei Torii*, Kaoruko Ohtani, Takahito Niwa, Naohiro Ishii \\ Aichi Institute of Technology, 1247 Yachigusa, Yakusa-Cho, Toyota, Aichi, Japan. \\ *Corresponding author. Tel.: +81-565-48-8121; email: mac@aitech.ac.jp \\ Manuscript submitted May 10, 2016; accepted December 17, 2016. \\ doi: $10.17706 /$ jcp.13.1.35-43
}

\begin{abstract}
In this study, the development of the objectivity index for the diagnosis of the autistic children with a lack of the communication ability and an evaluation of the curative effect using the ocular movement measurement is discussed. In past study, we developed communication applications "Eye Talk" and "Eye Tell" for people who have difficulty in conversation and writing such as children with physical disability, ALS patients or elderlies using the blink determination system. The team of Dr. Kitazawa in Graduate School of Frontier Biosciences in Osaka University performed the clinical application to distinguish autism group by measuring "where and when" he/she looks at using Tobii eye trucker. Our study is a judgment by the ocular movement measurement. We developed the image processing technique by afterimage used in the blink determination. First, the eye area is captured by a front camera of laptop PC. Second, we extracted the pixels of pupils with 30-40 fps of accuracy and digitized eyeball movements. We converted the difference in eyeball movements between the right and left eyes into a graph and define it in multidimensional measure. We measured the amount of the degree that the eyes of the subject run off the track based on the afterimage, then added up the amount of change of right and left eyes and showed the total. Finally, we set the identification border with density function of the distribution, cumulative frequency function, and ROC curve. With this, we established an objective index to determine autism, normal, false positive, and false negative. This method is used as an objective evaluation indicator to judge non-autistic and autistic people more clearly in early stage.
\end{abstract}

Key words: Oculomotor, autism, ROC curve, afterimage.

\section{Introduction}

This paper shows the development an assessment tool to diagnose autism by the measurement of eyeball movements. Autism is a developmental disability of communication and the social nature. The number of patients have been increasing, but the basic mechanism of the onset is not yet elucidated and effective cures or precautionary measures are not established [1]-[4]. In Japan, it is reported that approximately 10\% of boys and $4-5 \%$ of girls in primary schools are considered to have some developmental disabilities. Act on Support for Persons with Developmental Disabilities is passed in 2003, and the special support education to children with disabilities is started in 2006 [5], [6]. Also, the body of laws and administrative frameworks are maintained. Based on such background, an assessment tool to evaluate not only intellectual abilities and the individual developmental characteristic but also the adaptive behavior or the ability that is necessary to live in society are demanded with medical and welfare institutions to adopt to a real daily life. In addition, it is necessary to support children with developmental disabilities on a long-term basis from infants to adults. To 
detect and respond to disabilities in early stage, the assessment tool which everyone can give diagnoses is needed.

\section{Purpose of the Study}

In past study, we developed communication applications "Eye Talk" and "Eye Tell" for people who have difficulty in conversation and writing such as children with physical disability, ALS patients or elderlies using the blink determination system. These applications receive strong supports from not only special support schools in Japan but also some researchers in foreign countries [7]-[9]. Then we developed the image processing technique by afterimage used in the blink determination. First the eye area is captured by a front camera of laptop PC. Even when a person gazes at one point and his/her eyeballs always stay fixed at that point, their eyes perform subtle fixating movements (ie. tremors, drifting, microsaccades) to keep the retinal image clear. Particularly, the microsaccades link with nerves and reflect the mechanism that process sight in a brain. We converted the differences between these movements into numbers.

The process of the conversion is as followed:

(1) Select the pixel indicating the subject's pupil from images of captured frames.

(2) Set up a reference image, known as an afterimage, from the pixel indicating the subject's pupil.

(3) Divide the pupil of the subject into four from the center in the acquired frame image.

(4) Select the pixel in each divided part and count the number of the pixels of the overlapping part with the present pixel based on the afterimage.

(5) Process the images with precision in 30-40fps from a camera and convert the amount of change in the pixels of the movements of the right and left eyeballs in to numbers.

Then the pixels of pupils were extracted with 30-40 fps of accuracy and eyeball movements were digitized.

We converted the sequence of eyeball movements into a graph and define it in multidimensional measure. Then we set the identification border with density function of the distribution, cumulative frequency function, and ROC curve. With this, we established the objective index to determine autism, normal, false positive, and false negative. With this method, we developed the objective evaluation indicator to judge non-autistic and autistic people more clearly in early stage. By this new assessment, the degree of disabilities and the needed supports become clear. In addition, more accurate and suitable supports can be offered to each autistic children by considering the intelligence level or the degree of development. The additional evaluations including the possibility to jobs are enabled by grasping a merger of the mental disabilities and the psychoneurotic merger.

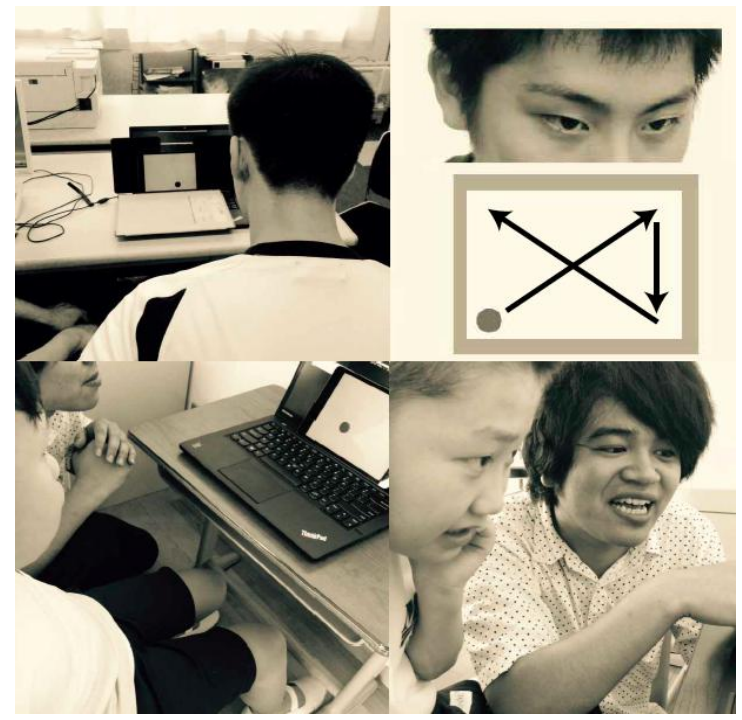

Fig. 1. Acquisition of eye movement of children with autism. 


\section{Structure of the System}

\subsection{Definition of Afterimage and Digitizing Oculomotor Change}

The difference in the area of the amount of change occurs by measuring the difference between the afterimage in consecutive frames and the present frame. We set the amount of change to the quantity of the eyeball movements. This method made it possible to detect a change of the eyeball movements in numerical value. We compared the difference in these movements between non-autistc and autistic people and analyzed the result.

The definition of the afterimage and the detection process are as follows.

When the area (C, Fig. 2) that is over-lapping part of the afterimage of iris (A, Fig. 2) and the cur-rent iris situation (B, Fig. 2) is diminishing rapidly comparing from a few frames before, we determine it as closing of the eyes. It is possible to exclude a slow change and capture a rapid change. By using the afterimage, the number of past frames to compare increases and we can capture changes with high accuracy. As a result, we can quantify the changes of series of movements "eye-opening $\rightarrow$ eye-closing $\rightarrow$ eye-opening". And it is possible to determine the blinks as short blinks, long blinks, or closing the eyes continuously, except the malfunction due to fine movement of eyes. Fig. 2 shows determination by afterimages visually. A is the afterimage to be compared, $\mathrm{B}$ is the iris in current frame, and $\mathrm{C}$ is the over-lapped area of the afterimage and current frame.

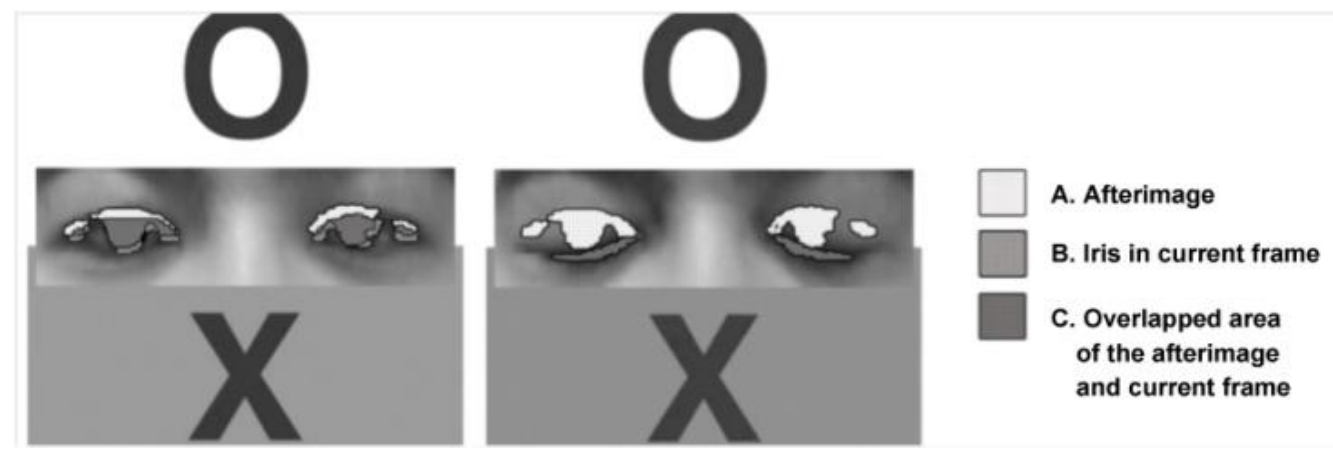

Fig. 2. Detection of unconcious blink by afterimages, visually.

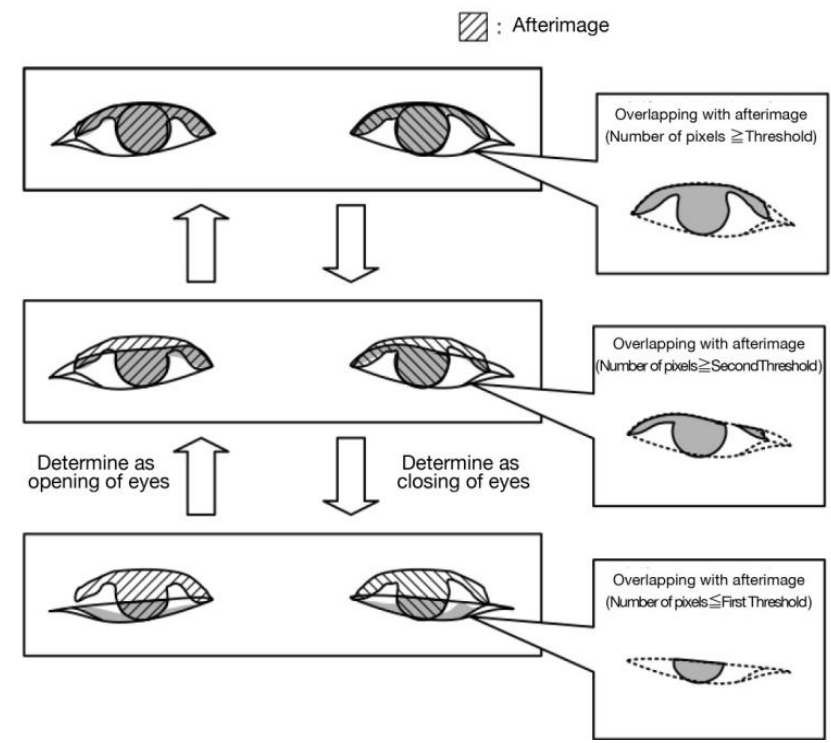

Fig. 3. Diagram of opening and closing of eye.

Fig. 3 is an illustration indicating the states to judge opening and closing of eyes. Since it is difficult for a subject with weak muscular strength to close his/her eyes perfectly, it may be with a half-shut blink even if it 
were to be a conscious blink, as shown in the middle and lower section of Fig. 3.

Fig. 4 is the illustration indicating of the relations with the number of frames and the blink judgment. Even if a subject has weak muscle strength, an unconscious blink (a blink carried out naturally) is faster than a conscious blink.

The number of the first frames $\mathrm{K} 1$ is set from a few frames to more than 10 and less than 20 frames to remove such a fast unconsciousness blink. In this way, we can remove the unconscious-ness blink from the closing and opening of eyes that continued in a few frames (Fig. 4(a)). On the other hand, the number of the second frames $\mathrm{K} 2$ is set from in dozens of frame degree (ex; 50 frames) to remove the blink that is slower than a nor-mal, consciousness blink. It makes it possible to cover a case where a subject with weak muscle strength continues closing his/her eyes because he/she becomes tired to watch a screen from conscious blinking (Fig. 4(b)). In this way, we remove an unconsciousness blink and the case that a subject continues closing his/her eyes and determine it as a conscious blink when $\mathrm{K}$ (the number of the frame acquisition) is in the range between $\mathrm{K} 1$ (the number of the first frame) and $\mathrm{K} 2$ (the number of the second frame) to judge a conscious blink appropriately. In addition, if the sensitivity in blink judgment sensitivity setting part 44 becomes higher (if it gets closer to value 100), K1 is set in a small value and K2 is set in a big value, and the appointed number range will increase. Then, the fast blink that is almost an unconsciousness blink is judged as a consciousness blink. For this issue, we can remove an unconsciousness blink and the continued closing of eyes properly by adjusting it to an appropriate set point.

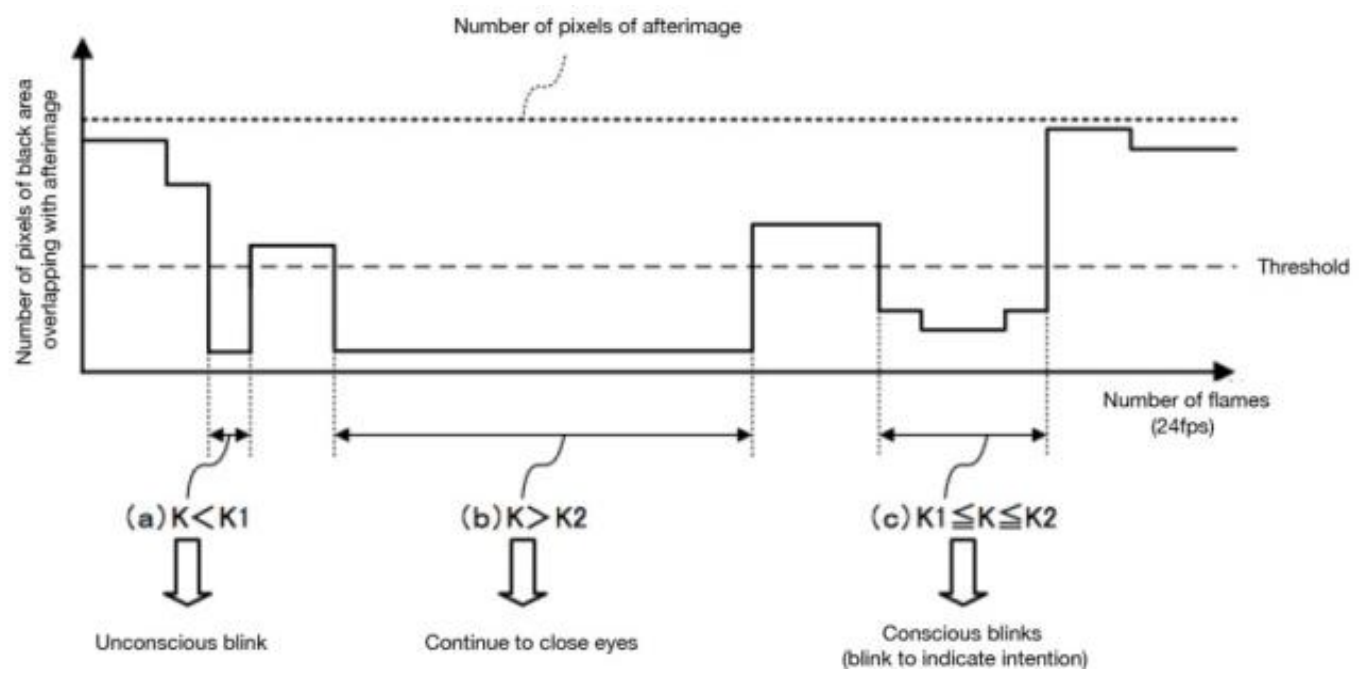

Fig. 4. Relationship between number of frames and blink determination.

The application "Eye Talk" which the selected letters are pronounced with is developed by using this highly precise blink judgment. We focused on making it possible to choose a letter less.

The definitions of the afterimage generation are as followed: After judging white or black, the part that is being recognized as black is determined to be an afterimage.

When it continues being recognized, it means that it is judged as black more than about $13 \mathrm{f}$ in the case of $24 \mathrm{fps}$ and it is deter-mined to be an afterimage.

Images are always judged consecutively. All pixels have Persistence Value and subtract 1 frame from every value.

In addition, Persistence Value does not become less than 0 and is always an integer. When it is determined that a pixel is black, 5 values are added.

We define the black part as an afterimage based on value of image persistences. The value becomes - 1+5 when the value of image persistences is judged to be black, and we make the program considering it as an afterimage when the value is over 50. Over 50 means the part which is judged as a black part during 
approximately 0.45 seconds is determined as an afterimage because 24-30 frames are processed per a second.

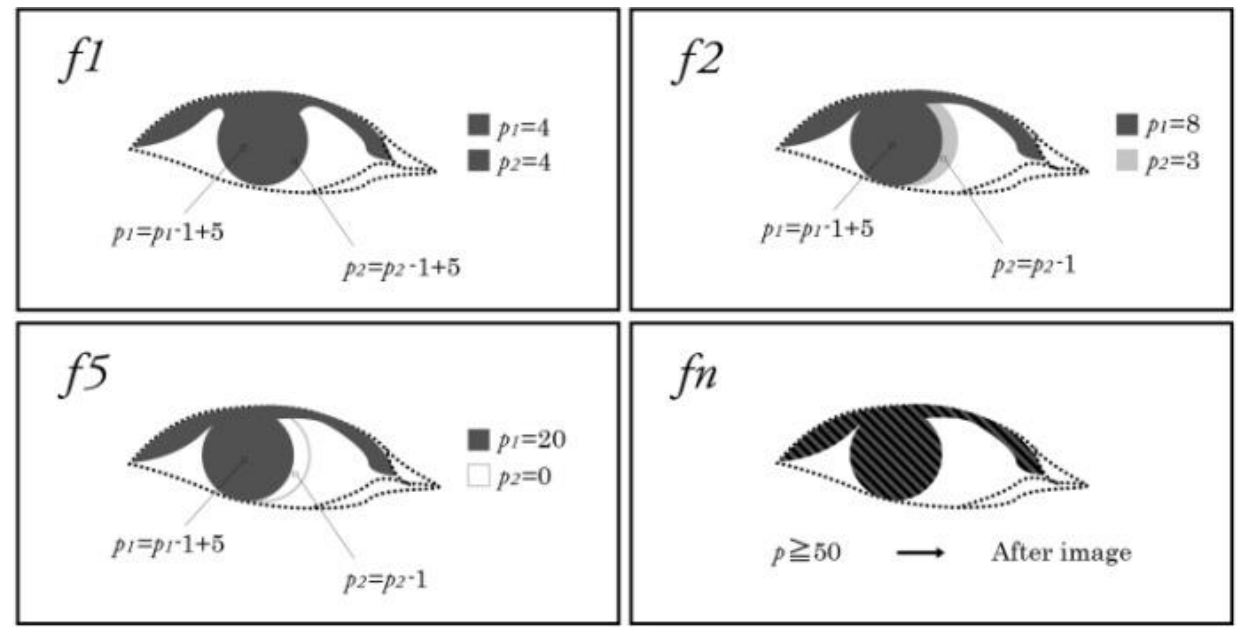

Fig. 5. The change of persistance value.

In the eye movement measurement with a tablet, the position sensing of the eyes part using the OpenCV HAAR-like assorter burdened the image processing.

Therefore we decided to use the image from the front camera of the laptop PC this time instead of a tablet. With parallel computation by PC multi-tasking, we can always demand ocular centers. We divided the iris of the eye into 4 parts from the center point and set the image persistence value. When the value is beyond "50 level", it assumes as an afterimage. We compare the number of the current pixels with the afterimage and determine it as eye movement in right and left when each condition is met. Fig. 12 shows the condition of each judgment.

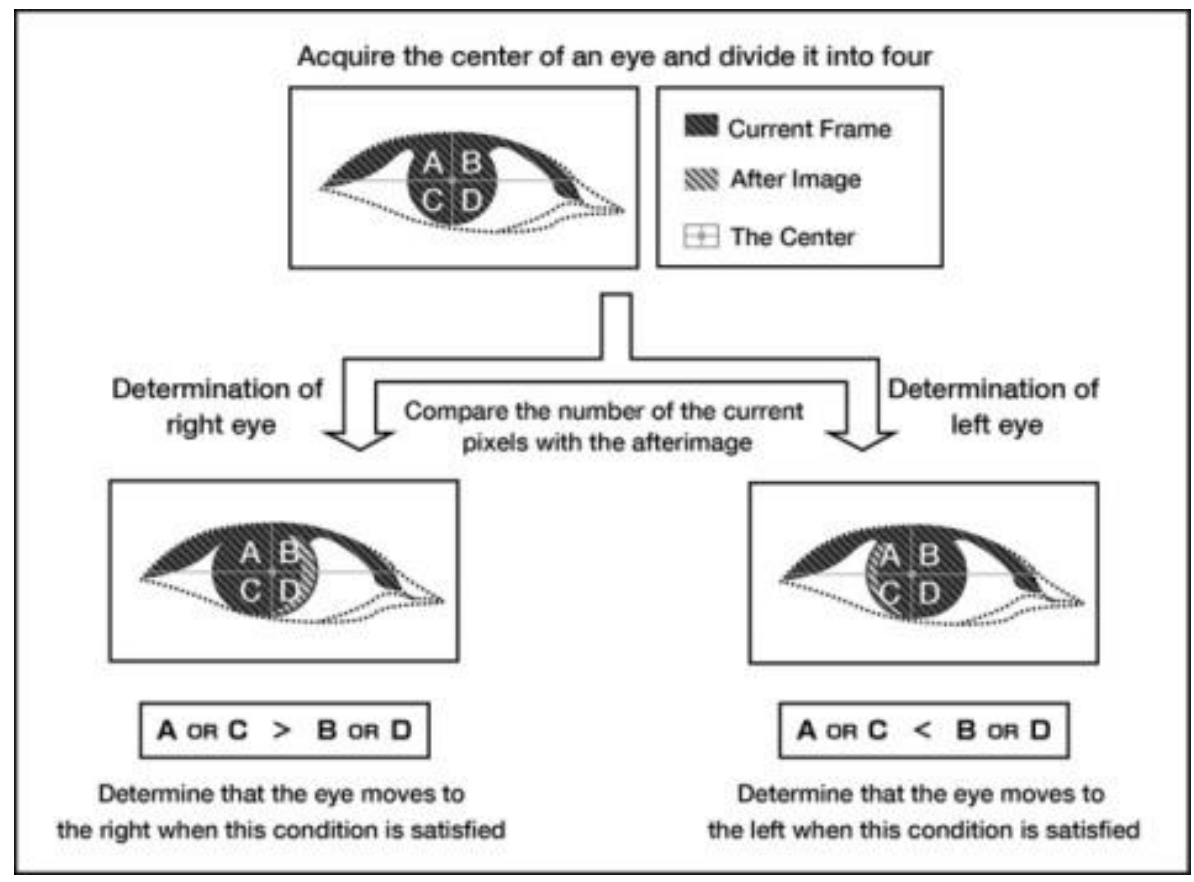

Fig. 6. Gaze direction detecting.

This blink determination and gaze detraction detecting make it possible to choose letters remarkably fast (Fig. 7).

We measured the amount of change of the eyeball movements of right and left eyes with precision of 
$14 \mathrm{fps}$ - 18fps by the comparison with the afterimage. The right part [Fig. 7] summarized the number of the pixels of right and left eyes divided in 4 in every 1 flame. We divide the right eye into number 1 to 4 , and the left eye into number 5 to 8 and digitize the number of each pixel.

(1)

(2)

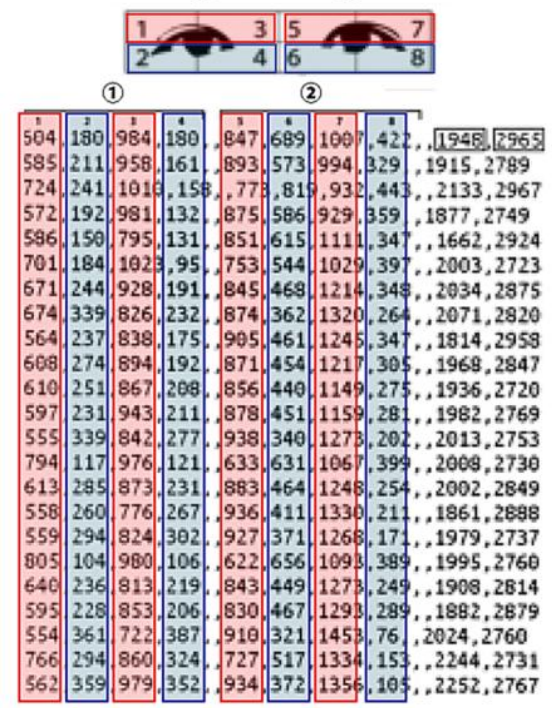

Fig. 7. Number of the pixels of afterimage divided into four parts.

\section{Clinical Application of the Subtle Eyeball Movements for Handicapped Children}

$\mathrm{U}$ The team of Osaka University discovered abnormality of the gaze activity of children with Attention Deficit Hyperactivity Disorder (ADHD) by measuring fast oculomotor movement (saccade ocular movement) using eye-tracking (Toby) in "Research trends in eye-hand coordination of mentally retarded persons" [10]-[13].

Diagnosis are made based on a comparison of "how long we look into the subject's eyes", that is the time of gazing an object in the conventional autistic study [14]-[16], but the results do not match.

We compared the autism group and the normal development group by measuring pursuit with the eyeball movement measuring device. Our research subjects consists of 7 children and 15 adults with autism, and 7 children and 15 adults with no disability.

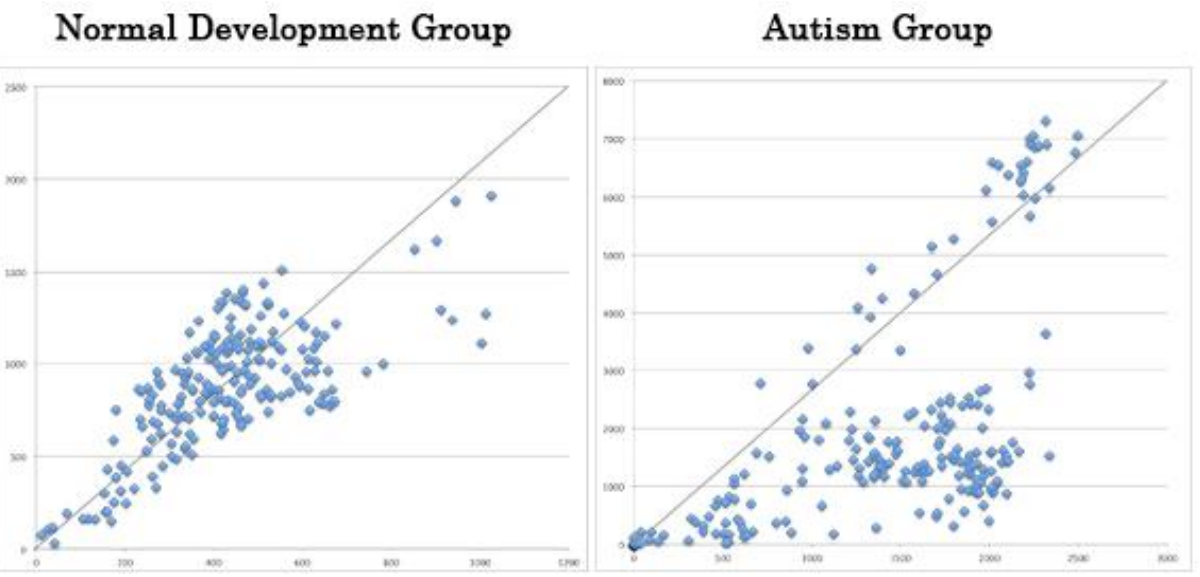

Fig. 8. Difference of eyes dispersion between normal development group and autism group.

Pursuit is a smooth movement of eyes when a subject keep watching a target which moves slowly (15fps) with the fovea. We measured the amount of the degree that the eyes of the subject run off the track based on the afterimage, then added up the amount of change of right and left eyes and showed the total. Fig. 8 is the 
distribution map which showed the oculomotor dispersion of each group.

While the dots were concentrated in the center line in the normal development group, they were scattered around the area in the autism group. It indicate that subjects in the normal development group can follow pursuit the moving target with eyes, but gaze of subjects in the autism group run off the track easily. By comparing the distance from the center line of this distribution in each group, it is proved that the distance of the autism group is significantly farther than the normal development group in both children and adults.

Fig. 9 shows the relations of the normal development group and the autism group by the function of density of probability.

The abscissa of the Fig. shows the amount of change of the pixels in the both groups.

The amount is distributed according to the average 113 and the standard deviation 36 in the normal development group, while the average is 590 and the standard deviation is 200 in the autism group. The ordinate shows the density of probability.

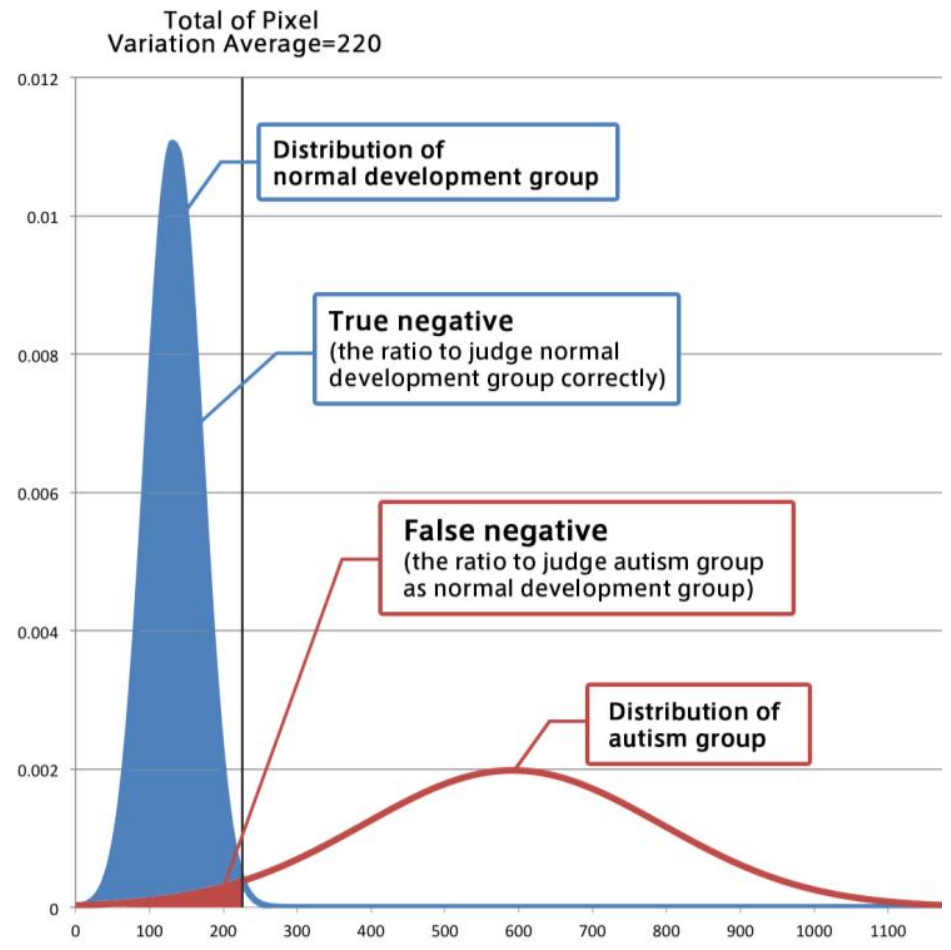

Fig. 9. Relations of normal development group and the autism group with the density of probability.

Table 1. Boolean table indicating whether positive or negative

\begin{tabular}{|c|c|c|}
\hline & FALSE & TRUE \\
\hline $\begin{array}{c}\text { Negative } \\
(220<)\end{array}$ & false negative rate & $\begin{array}{c}\text { true negative rate } \\
0.99\end{array}$ \\
\hline $\begin{array}{c}\text { Positive } \\
(\leq 220)\end{array}$ & $\begin{array}{c}\text { false positive rate } \\
0.01\end{array}$ & $\begin{array}{c}\text { true positive rate } \\
0.96\end{array}$ \\
\hline
\end{tabular}

The decision surface line is the point of intersection of the density of probability of each group. From a graph, we set it as the decision surface line when the total amount of change of the pixels is 220 .

The true negative rate that could judge the normal development group is $99 \%$ and the false positive rate is $1 \%$ (Table 1).

The truth positive rate to judge the autism group is $96 \%$ and the false negative rate that judges an autistic person as normal (Table 1). 
We judged the performance evaluation of this measurement with ROC curve (Fig. 10) [17]. On ROC, the shape shifts to the top left when there is little heap of two density functions, and shows that the performance of the decision surface line is high. AUC(Area under the curve) surrounded by the ROC curves is AUC = 0.9868 . Since the area is big, it is considered that the reliability of this method is high.

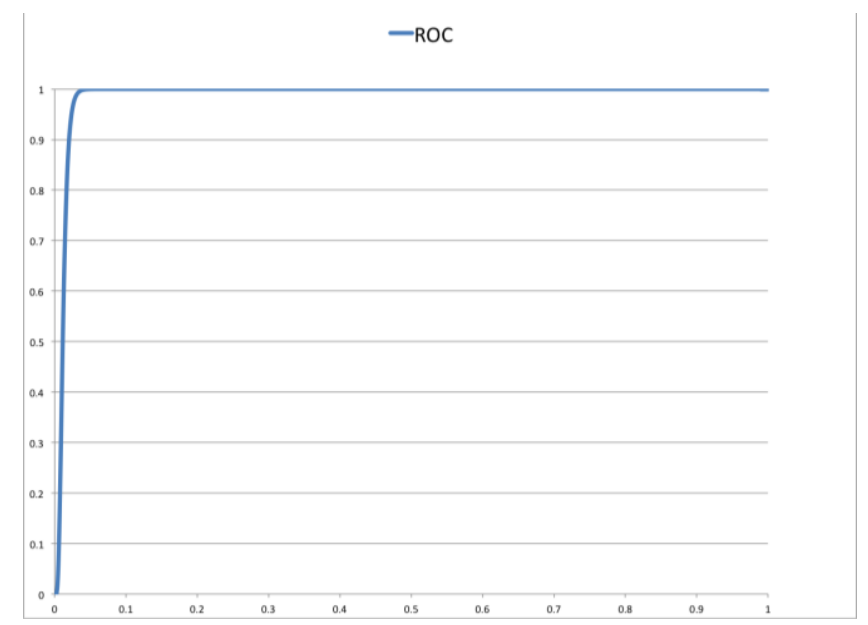

Fig. 10. ROC curve showing the accuracy of the judgment ; AUC=0.9868.

\section{Conclusion}

In this study, it was intended to establish the objective index using the ocular movement to be used for the diagnosis of autistic children based on our past researches. We succeeded to develop the method of the ocular movement measurement with highly precise. By using the front camera of the popular laptop PC, we established the objective index to judge the autism group, the normal development group, the truth negative and the false negative without using an expensive devise.

This method can carry out important tasks as the supporting assessment tool of the judgement of psychiatrists to discover disabilities in children and consider appropriate correspondence. In addition, as well as a judgment, it is considered that this method leads to evaluations of education and curative effects in educational fields.

\section{References}

[1] Kitazawa, S. (October2005). "Probabilistic mechanisms of learning and development in sensorimotor systems" ESF-EMBO symposia on "Three-Dimensional Sensory and Motor Space". Where Tactile Stimuli Are Ordered in Time. San Feliu de Guixols, Spain.

[2] Kitazawa, S. (2006). Reversal of subjective temporal order due to sensory and motor integrations. Attention and Performance XXII. Sensorimotor Foundations of Higher Cognition. Chateau de Pizay, France.

[3] Kitazawa, S. (2007). Reversal of subjective temporal order due to eye and hand movements. ESF-EMBO symposia on "Three-Dimensional Sensory and Motor Space". SantFeliu de Guixols, Spain.

[4] Kitazawa, S., \& Nishida, S. (2008). Adaptive anomalies in conscious time perception. Tutorial workshop in the Proceedings of 12th Annual Meeting of the Association for the Scientific Study of Consciousness. Taipei, Taiwan.

[5] Ministry of Health, Labour and Welfare. (2013). Guidelines about person of development child with a disability support and the assessment.

[6] Guidelines about person of development child with a disability support and the assessment. (2012).

[7] Torii, I., Ohtani, K., Niwa, T., \& Ishii, N. (2015). Detecting eye-direction using afterimage and ocular movement. Proceedings of ACIS 2nd International Symposium on Computational Science \& Intelligence (pp. 149-154).

[8] Torii, I., Ohtani, K., Niwa, T., \& Ishii, N. (2015). System and method for detecting gaze direction. Proceedings of 
The 6th International Conference on Information, Intelligence, System and Applications.

[9] Torii, I., Ohtani, K., Niwa, T., \& Ishii, N. (2015). Verbal communication aid application with eye-blink. Proceedings of WCCAIS' International Conference on Computer Information Systems (pp. 296-302).

[10] Nakano, T., Tanaka, K., et al. (May 19, 2010). Atypical gaze patterns in children and adults with autism spectrum disorders dissociated from developmental changes in gaze behaviour. Proceedings of $R$ Soc B. (pp. 2935-2943).

[11] Kitazawa, S. Eye Tracking Research. From http://www.tobii.com/Global/Analysis/Marketing/JapaneseMarketingMaterial/CustomerCases_JP/Tobii_Cu stomerCase_Clinical_application_and_eye_tracking_gaze_tracking_in_autism.pdf

[12] Nakano, T., Ota, H., Kato, N., Kitazawa, S. (Apr. 7, 2010). Deficit in visual temporal integration in autism spectrum disorders. Proceedings of R Soc B. (pp. 1027-1030).

[13] Kitazawa, S. (2010). Towards the understanding and treatment of autism. Series Mobiligence, 4, 213-54.

[14] Kimura, Y., \& Kobayashi, H. Clinical Study on movement education application of autistic children - approach to enhance the Motor Imitation. Proceedings of the 25th Japan Special Education Society Papers (pp. 446-447).

[15] Iwanaga, R., \& Kawasaki, C. For sensorimotor disorder of high- functioning autism children, children of the spirit and nerve. 36(4), 27-332.

[16] Fukuda, S., Okamoto, M., Kato, K., Murata, E., Yamamoto, T., Mohri, I., \& Taniike. M. (2011). A perception of the others' gaze for children with pervasive developmental disorders. Human Developmental Research, 25, 135-148.

[17] Kawashima, H., Hayashi, N., Ohno, N., Matsuura, Y., \& Sanada, S. (2015). Comparative study of patient identifications for conventional and portable chest radiographs utilizing ROC analysis. Japanese Society of Radiological Technology, 71(8), 663-669.

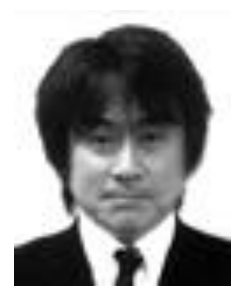

Ippei Torii received B.F.A. degree in sculpture of fine arts from Nihon University, Tokyo, Japan, in 1982. From 1982 to 1984 he was at the School of Fine Arts in Nihon University. And he received Ph.D degree in management information science from Aichi Institute of Technology in 2012. From 2015 he has been an professor in Aichi Institute of Technology. His research interests include computer graphics, HCI and visual design. He is a member of IEEE, ACIS and Design Research Association.

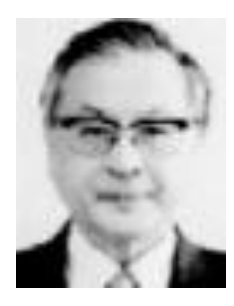

Naohiro Ishii received the BE and ME degrees and the Ph.D degree in electrical communication engineering from Tohoku University, in 1963, 1965 and 1968, respectively. He was a professor in Computer Science and Engineering, Nagoya Institute of Technology and in Information Science, Aichi Institute of Technology, respectively. He is a visiting professor in Information Science, Aichi Institute of Technology. His research interest includes computer system, artificial intelligence, data mining and web applications. He is a member of IEEE , ACM and ACIS. 